\title{
Blutgefässsegmentation in MR-Bildern mittels Aktivkontur-Modellen
}

\author{
Kozerke, S ; Botnar, R ; Scheidegger, M B ; Boesiger, P
}

DOI: https://doi.org/10.1515/bmte.1996.41.s1.636

Posted at the Zurich Open Repository and Archive, University of Zurich ZORA URL: https://doi.org/10.5167/uzh-155077

Journal Article

Published Version

Originally published at:

Kozerke, S; Botnar, R; Scheidegger, M B; Boesiger, P (1996). Blutgefässsegmentation in MR-Bildern mittels Aktivkontur-Modellen. Biomedizinische Technik. Biomedical engineering, 41(s1):636-637.

DOI: https://doi.org/10.1515/bmte.1996.41.s1.636 


\title{
Blutgefässsegmentation in MR-Bildern mittels Aktivkontur-Modellen
}

\author{
Kozerke S., Botnar R., Scheidegger M.B., Boesiger P. \\ Institut für Biomedizinische Technik und Medizinische Informatik \\ Universität und ETH, CH-8044 Zürich, Schweiz
}

\section{EINLEITUNG}

Die Untersuchung der Strömungsverhältnisse des kardio-vaskulären Systems und die Berechnung von Flussparametern in Blutgefässen mittels MRI-Methoden erfordert die Bestimmung der Blutgefässbegrenzungen in einer zeitlichen Abfolge von phasenkodierten Bildern. Die manuelle Kennzeichnung der Gefässkonturen mit graphischen Eingabehilfen ist sehr zeitaufwendig und deren Genauigkeit vom Benutzer abhängig. Um die Konturdetektion der Gefässquerschnitte effektiver zu gestalten wurde ein Softwarepaket, basierend auf Aktivkonturen [1], entwickelt. Aufgrund von möglichen Bildartefakten in den Intensitätsbildern (Modulus) während der Systole wird die Phaseninformation (Phase) ebenfalls für die Segmentation genutzt.

\section{METHODEN}

Grosse Arterien (z.B. Aorta asc.) können sich beträchtlich in Form und Position während eines Herzzyklus' verändern. Deshalb wird die Veränderung des Gefässquerschnitts in eine Translations- und eine Deformationskomponente zerlegt (Figur 1).

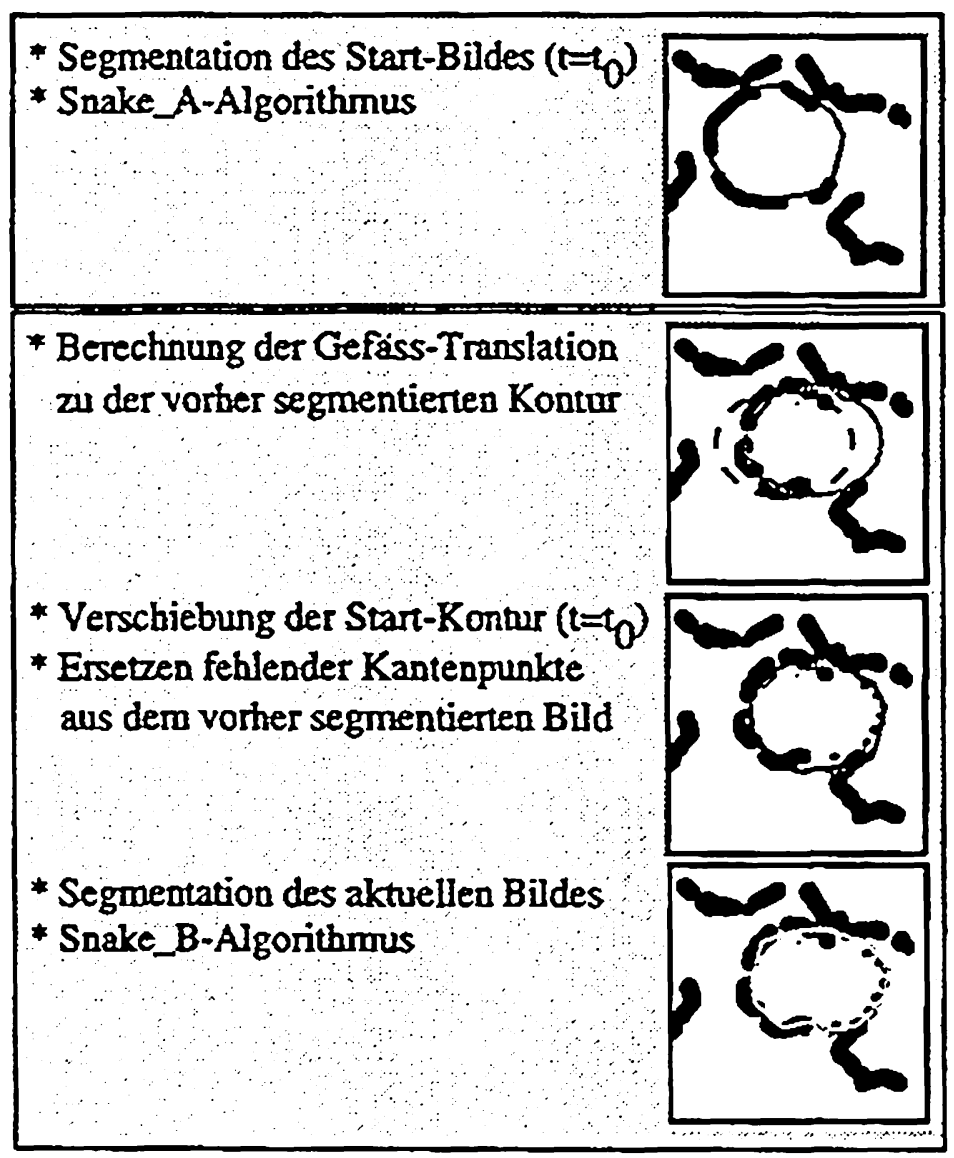

Figur 1: Die Segmentation der Gefässquerschnitte erfolgt durch Bestimmung einer Translations- und einer Deformationskomponente; die Startbildkontur wird als Ähnlichkeitsreferenz genutzt, um zu starke Abweichungen der Gefässformen zu verhindern
Die Translation bestimmt die ungefähre Verschiebung des Gefässquerschnitts zwischen zeitlich benachbarten Herzphasen. Die Verschiebung wird durch eine Kreuzkorrelationsmethode ermittelt. Die Detektion der Formänderung des Gefässquerschnitts erfolgt durch Anwendung von Aktiv-Kontur-Algorithmen (Figur 2).

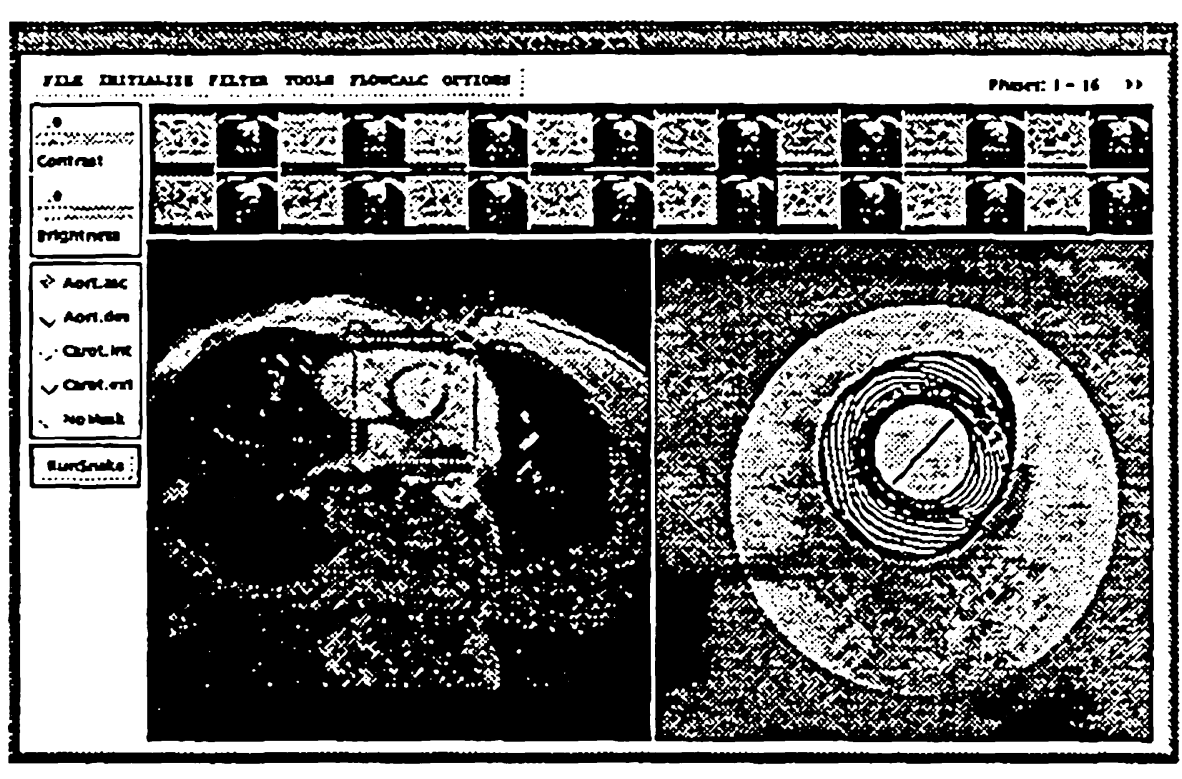

Figur 2: Bestimmung der Deformation des Gefässquerschnitts durch einen Aktivkontur-Algorithmus ausgehend von einer Initialkontur (Benutzer setzt 2 Punkte innerhalb des Gefässes, die durch eine Splinefunktion verbunden werden)

Querströmungen innerhalb des Gefässquerschnitts und Bewegungen höherer Ordnung führen während der Systole führen oft $\mathrm{zu}$ Bildartefakten in den Modulusbildern. Die direkte Auswertung der Modulusinformation zu Zeitpunkten der Systole führt zu unzuverlässigen Ergebnissen in der Konturdetektion. In diesen Fällen werden die Phasenbilder für die Segmentation herangezogen. Die gesamte Vorgehensweise zur Segmentation benötigt a priori Wissen, z.B. Parameter für maximale Verschiebung und Grössenänderungen der Gefässe während des Herzzyklus'. Diese Parameter werden mit der Angabe der zu untersuchenden Gefässart durch den Benutzer eingestellt. Ein Bild der Bildserie wird automatisch anhand der Grauwertvarianz als Startbild ausgewählt. Der Benutzer setzt auf diesem Bild durch 2-3 Punkte eine beliebige Initialkontur innerhalb des zu untersuchenden Gefässes, da ausserhalb des Gefässes Kanten anderer Organe zu einer fehlerhaften Segmentation führen können. Ausgehend von dieser Kontur detektiert ein Aktivkonturalgorithmus (Snake_A) die Gefässgrenzen. Um eine Bewegung der Aktivkontur ausgehend von einer Initialposition innerhalb des Gefässes nach aussen zu den zu suchenden Gefässgrenzen zu bewirken, wird ein in [2] vorgestelltes 
Balloon-Modell genutzt. Die ermittelte Kontur des Startbildes wird als Ähnlichkeitsreferenz gespeichert. Diese Referenz verhindert bei der Segmentation aller weiteren Herzphasen ein Anheften der Aktivkontur an Kanten möglicher Bildartefakte oder angrenzender Organe. Hierzu wird die Kontur des Startbildes als Schablone bei der Segmentation aller weiteren Bilder der Zeitserie genutzt. Die Detektion der Gefässbegrenzungen erfolgt sequentiell, ausgehend vom Startbild (Snake_A), durch den zweiten AktivkonturAlgorithmus (Snake_B). Den Segmentationsablauf zeigt Figur 3.

\begin{tabular}{|c|c|}
\hline \multirow[t]{4}{*}{ Initialisierung } & $\begin{array}{l}\text { Benutzer-Eingaben } \\
\text {-Region of Interest (ROI) } \\
\text {-Gerásstyp }\end{array}$ \\
\hline & Unierdrickung Zufallsphase \\
\hline & Bestimmung Starbild \\
\hline & $\begin{array}{l}\text { Benutzer-Eingabe } \\
\text {-Eingabe Starkontur }\end{array}$ \\
\hline \multirow[t]{3}{*}{ Segmentation des Startbildes } & Snake_A-Algorithmus \\
\hline & $\frac{\text { Benutzer-Ėingabe (optional) }}{\text {-Kontur-Korrektur }}$ \\
\hline & Sichere Kontur als Referenz \\
\hline \multirow[t]{2}{*}{$\begin{array}{l}\text { Segmentation aller anderen } \\
\text { Phasen des Herzyylus }\end{array}$} & Kanten+Kontur-Propagierung \\
\hline & Snake_B-Algorithmus \\
\hline
\end{tabular}

Figur 3: Programmablauf zur Segmentation der Gefässbegrenzungen einer zeitlichen Abfolge von Bildern; die Segmentation pro Herzphase erfolgt entweder auf dem Modulus- oder Phasenbild in Abhängigkeit der Bildqualität

\section{ERGEBNISSE}

Die Segmentation der Carotis int. und der Aorta asc. erfolgte einerseits automatisch durch die vorgestellte Software und andererseits manuell durch einen erfahrenen Benutzer.
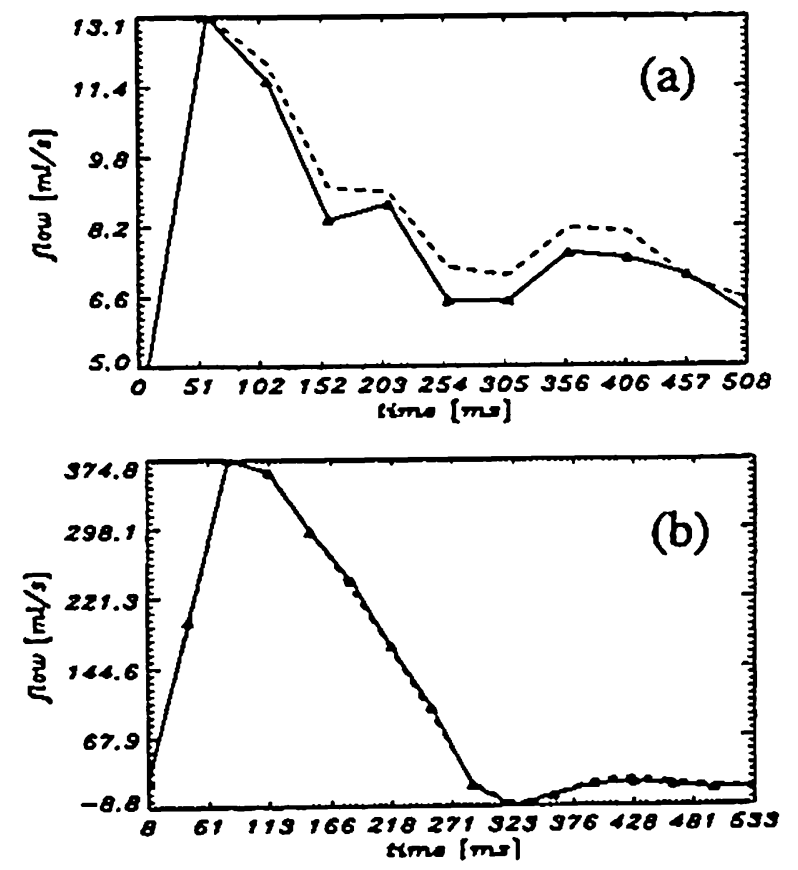

Figur 4: Mittlerer Blutflussverlauf der Carotis int. (a) und der Aorta asc. (b) berechnet aus der automatisch erfolgten Segmentaion $(-)$ und manuell markierten Konturen (--)
Den Vergleich der mittleren Blutflussverläufe der Carotis int. (a) und der Aorta asc. (b) zeigt Figur 4.

Um die Genauigkeit der vorgestellten Segmentation zu beurteilen wurden 8 Probanden und zwei Patienten mit einem mechanischen Klappenersatz untersucht. Die Geschwindigkeitsverteilungen der Aorta asc. wurden ungefähr $30 \mathrm{~mm}$ stromabwärts der Aortenklappe aufgenommen. Die Aufnahmen erfolgten auf einem Philips NT 1.5 Tesla Tomographen mittels einer Herzspule. Die lineare Regression der aus den segmentierten Geschwindigkeitsbildern berechneten Auswurf-Volumina der Probandenstudie ist in Figur 5 dargestellt.

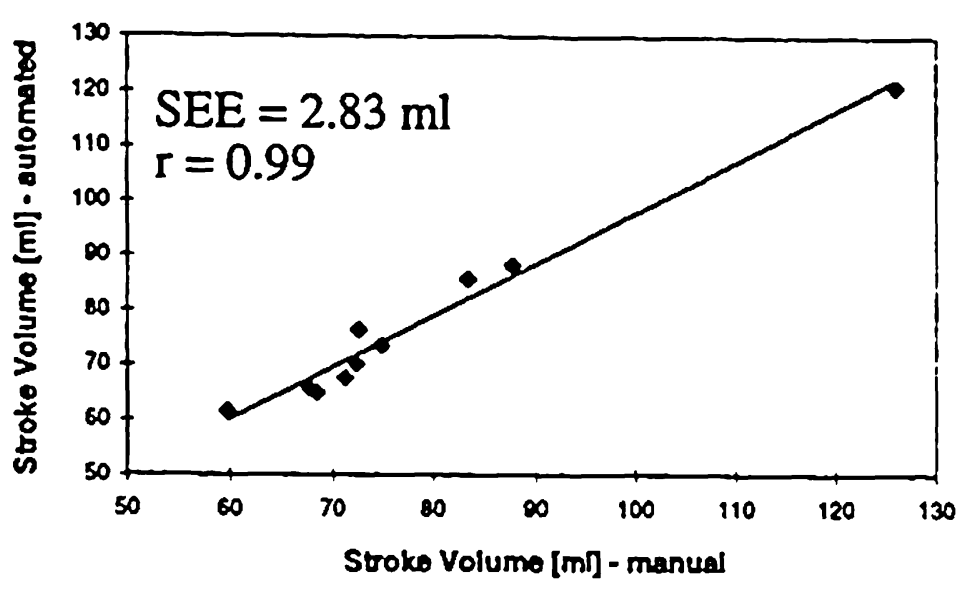

Figur 5: Lineare Regression der Auswurf-Volumina (Stroke Volume) berechnet aus Geschwindigkeitsbildern mit manuell und automatisch ermittelten Gefässkonturen

Die Implementierung der Segmentationsalgorithmen erfolgte mit dem Signal-Processing-Package PV-WAVE (Visual Numerics Inc.) für UNIX als auch VMSSysteme geeignet. Für die Segmentation und Berechnung der mittleren Blutflusskurven liegt der Zeitaufwand im Minutenbereich. Eine manuelle Segmentation durch einen erfahrenen Benutzer erfordert ungefähr die 5-fache Zeit.

\section{SCHLUSSFOLGERUNGEN}

Die gute Übereinstimmung der bestimmten Volumina der automatisch und der manuell bestimmten Konturen der Aorta asc. demonstriert die Zuverlässigkeit der vorgestellten Methode für die Untersuchung grösserer Arterien. Die Verwendung von Bereichen der Phasenbildkanteninformation sichert eine ungefähre Segmentation der Querschnitte auf Bildern, die aufgrund starker Blutströmungen in der Querschnittsebene Artefakte in den Modulusbildern aufweisen. Untersuchte Zeitserien kleinerer Arterien (z.B. Carotis int.) zeigen aufgrund der Abbildung eines solchen Gefässes durch eine geringere Anzahl von Pixeln grössere Ungenauigkeiten in der Bestimmung des mittleren Blutflussverlaufs.

\section{LITERATUR}

[1] Kass M., Witkin A., Terzopolous D., Proc. ICCV, IEEE, 259-268, 1987

[2] Cohen L.D., Cohen I., IEEE TPMI, Vol. 15, 11311147, 1993 